\title{
Foveal Sparing in Stargardt Disease
}

\author{
Ramon A. C. van Huet, ${ }^{1}$ Nathalie M. Bax ${ }^{1}$ Sarah C. Westeneng-Van Haaften,${ }^{1}$ \\ Muhamad Muhamad, ${ }^{1}$ Marijke N. Zonneveld-Vrieling, ${ }^{2}$ Lies H. Hoefsloot, ${ }^{2}$ Frans P. M. Cremers, ${ }^{2,3}$ \\ Camiel J. F. Boon, ${ }^{1,4}$ B. Jeroen Klevering, ${ }^{1}$ and Carel B. Hoyng ${ }^{1}$
}

${ }^{1}$ Department of Ophthalmology, Radboud University Medical Center, Nijmegen, The Netherlands ${ }^{2}$ Department of Human Genetics, Radboud University Medical Center, Nijmegen, The Netherlands ${ }^{3}$ Nijmegen Center of Molecular Life Sciences, Radboud University Medical Center, Nijmegen, The Netherlands ${ }^{4}$ Department of Ophthalmology, Leiden University Medical Center, Leiden, The Netherlands

Correspondence: Carel B. Hoyng, Department of Ophthalmology (400), Radboud University Medical Center, P.O. Box 9101, $6500 \mathrm{HB}$ Nijmegen, The Netherlands; Carel.Hoyng@Radboudumc.nl.

RACvH and NMB contributed equally to the work presented here and should therefore be regarded as equivalent joint first authors.

Submitted: December 23, 2013 Accepted: October 6, 2014

Citation: van Huet RAC, Bax NM, Westeneng-Van Haaften SC, et al. Foveal sparing in Stargardt disease. Invest Ophthalmol Vis Sci. 2014;55:7467-7478. DOI:10.1167/ iovs.13-13825
Purpose. To provide a clinical and genetic description of a patient cohort with Stargardt disease (STGD1) with identifiable foveal sparing.

Methods. Patients with retinal atrophy (defined as an absence of autofluorescence) that surrounded the fovea by at least $180^{\circ}$ and did not include the fovea were defined as having foveal sparing; eyes with visual acuity (VA) worse than 20/200 were excluded. We reviewed the medical files and extracted data regarding medical history, VA, ophthalmoscopy, static perimetry, fundus photography, spectral-domain optical coherence tomography (SD-OCT), fluorescein angiography (FA), fundus autofluorescence (FAF), and electroretinography (ERG). We screened each patient's $A B C A 4$ gene for mutations.

RESults. Seventeen eyes with foveal sparing were identified in 13 unrelated patients. In 4 eyes, the fovea gradually became atrophic after the initial foveal sparing. The mean age at onset was 51 years (range, 32-67 years). Visual acuity was 20/40 or better in all foveal sparing eyes and was $20 / 25$ or better in $41 \%$. Fundus autofluorescence imaging revealed hyperautofluorescent flecks and parafoveal retinal atrophy; SD-OCT revealed sharply delineated atrophy; and perimetry revealed parafoveal scotomas with intact foveal sensitivity. Finally, genetic screening identified mutations in 19 of the 26 ABCA4 gene alleles.

Conclusions. Foveal sparing occurs mainly in patients with late-onset STGD1 and represents the milder end of the clinical spectrum in STGD1. The anatomy, metabolism, and biochemistry of the retina, as well as genetic variations in genes other than $A B C A 4$, can influence the etiology of foveal sparing. Identifying these fovea-protecting factors will facilitate the future development of strategies designed to treat STGD1.

Keywords: foveal sparing, Stargardt disease, $A B C A 4$
$\mathrm{W}$ ithin the retina, the macula provides the highest visual acuity and contains the highest density of cones. ${ }^{1,2}$ Therefore, a loss of central vision is a hallmark feature of macular dystrophies. There are, however, exceptions to this rule. Foveal sparing is an intriguing phenomenon in which retinal atrophy surrounds a relatively preserved fovea, leaving central visual acuity largely unaffected. Although foveal sparing has been reported in a variety of conditions, including Stargardt disease (STGD1), ${ }^{3-5}$ mitochondrial retinal dystrophy associated with the m.3243A $>\mathrm{G}$ mutation, ${ }^{6}$ and geographic atrophy in age-related macular degeneration (AMD), ${ }^{7-11}$ its etiology remains poorly understood.

Stargardt disease is an autosomal recessive retinal dystrophy that typically presents within the first two decades of life. ${ }^{12}$ Although the clinical presentation of STGD1 varies widely, it is usually characterized by a progressive loss of central vision, irregular yellow-white fundus flecks, and the so-called beaten bronze atrophic macular lesions. ${ }^{13-15}$ Stargardt disease has been linked to mutations in the $A B C A 4$ gene, which encodes an adenosine triphosphate (ATP)-binding cassette transporter (ABCR) expressed specifically in the cones and rods of the retina. ${ }^{16,17}$ Defects in ABCR function cause the accumulation of all-trans-retinal and its cytotoxic derivatives (e.g., diretinoid- pyridinium-ethanolamine) in photoreceptors and retinal pigment epithelial (RPE) cells, ultimately causing RPE cell death and the subsequent loss of photoreceptors. ${ }^{18}$

Mutations in $A B C A 4$ have been linked to a spectrum of phenotypes ranging from mild macular dystrophy to severe early-onset panretinal dystrophy. ${ }^{3,19-22}$ We previously postulated that disease severity may be correlated with the functional severity of the particular mutation in the resulting ABCR protein. ${ }^{19,23-25}$ The substantial clinical variability among patients with STGD1-including an age at onset of the symptoms that can range from 5 to 72 years of age, diverse fundoscopic features, diverse electrophysiological findings, and a variable time course of vision loss-suggests the presence of several strong modifying factors. $3,13,14$

Recently, Fujinami et al. ${ }^{26}$ reported the clinical and molecular genetic findings of a cohort of STGD1 patients with relatively preserved foveal structure and function (based on seemingly normal autofluorescence at the fovea). Their study revealed the presence of two basic-yet distinct-STGD1 phenotypes, namely STGD1 patients with foveal sparing and STGD1 patients with early-onset foveal atrophy. ${ }^{26}$ However, the onset of foveal involvement in STGD1 can vary substantially and can occur in later disease stages, for example, in late-onset 


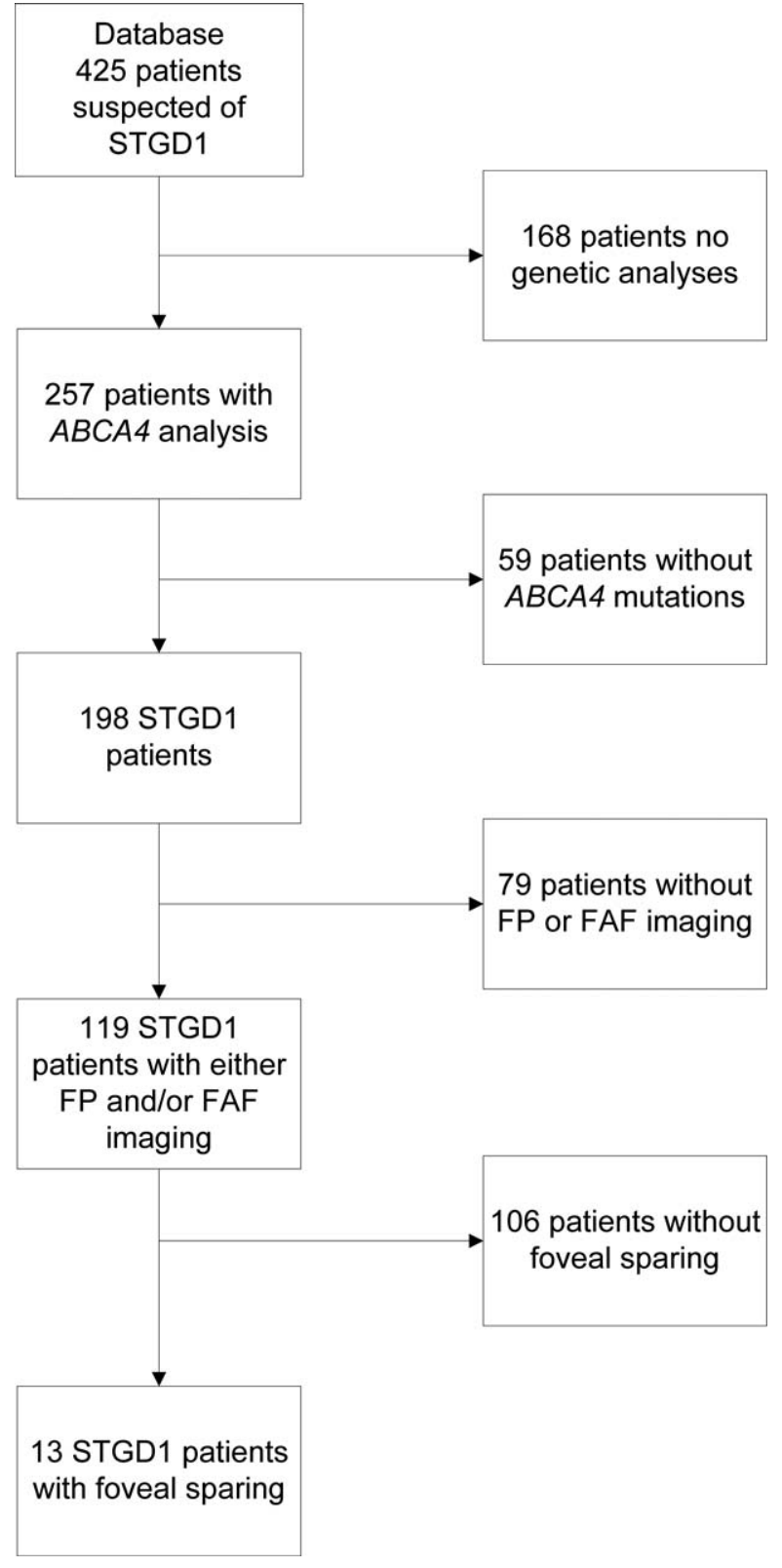

Figure 1. Flow chart depicting the selection process of Stargardt patients with foveal sparing for inclusion in the study. FP, fundus photography.

STGD $1 .{ }^{3}$ This heterogeneity complicates the selection of homogeneous cohorts for clinical studies to investigate the subtype of STGD1 patients with foveal sparing.

Here, we report the clinical characteristics and the natural course of foveal sparing in a cohort of STGD1 patients with foveal sparing, and we explore the mechanisms that may underlie this phenomenon.

\section{Methods}

\section{Patients and Genetic Analysis}

The patient selection process is depicted in Figure 1. The database of the Department of Ophthalmology at Radboud University Medical Center (Nijmegen, The Netherlands) contains 425 clinically suspected cases of STGD1. For 257 of these patients, an $A B C A 4$ genetic screen for known mutations was performed in the Department of Human Genetics at Radboud University Medical Center using arrayed primer extension analysis (APEX) microarrays (Asper Biotech, Tartu, Estonia). Because STGD1 is autosomal recessive, if the Asper microarray screen revealed only one mutation in a given patient, we sequenced the exons and intron-exon boundaries in $A B C A 4$ to identify the mutation in the second allele. All mutations were confirmed using Sanger sequencing. The presence of one or two mutations in the $A B C A 4$ gene confirmed the diagnosis of STGD1 in 198 patients. For our study, we selected cases in which foveal sparing was documented using fundus photography and/or fundus autofluorescence (FAF) imaging. Patients with RPE atrophy (defined as an absence of autofluorescence surrounding the fovea by least $180^{\circ}$ ) that did not include the fovea were defined as having foveal sparing. Eyes with visual acuity of 20/200 or worse were presumed to have little or no foveal function and were therefore excluded. Twelve unrelated STGD1 patients with foveal sparing in at least one eye were included in this study. We also included one additional patient who initially presented with foveal sparing; in this patient, the fovea became atrophic as the disease progressed. To exclude pseudoStargardt pattern dystrophy, ${ }^{27}$ the $P R P H 2$ gene was sequenced in all 13 patients.

This study was performed in accordance with the tenets of the Declaration of Helsinki, and all participating patients gave their informed consent prior to providing a blood sample and receiving additional ophthalmologic examinations.

\section{Clinical Examination}

Clinical data were collected from the medical records of the 13 eligible patients. The data collected included the patient's age at onset, medical history, initial symptoms, and the overall course of the retinal disorder. Age at onset was defined as the age at which the initial symptoms were noted by the patient. We defined the duration of symptomatic disease as the time from the age at onset to the patient's current age. In the patients who were initially asymptomatic, the age at their first visit to the ophthalmologist was used to define disease duration.

The standard ophthalmic examination included a measurement of best-corrected visual acuity (BCVA) using Snellen visual acuity charts and ophthalmoscopy. The central visual field was assessed with a Humphrey perimeter (Carl Zeiss Meditec, Jena, Germany) using central 10-2, 24-2, or 30-2 threshold tests in two, two, and four patients, respectively. Fundus photography (Topcon TRC50IX; Topcon Corporation, Tokyo, Japan) was performed in 10 patients. Fluorescein angiography (FA) was performed in 10 patients to screen for the presence of the dark choroid sign. Full-field electroretinography (ffERG; 8 patients) and multifocal ERG (mfERG; 7 patients) were performed using Dawson-Trick-Litzkow (DTL) electrodes and the RETI-port system (Roland Consults, Stasche \& Finger $\mathrm{GmbH}$, Brandenburg an der Havel, Germany). Both the ffERG and mfERG recordings were performed in accordance with the guidelines of the International Society for Clinical Electrophysiology of Vision (ISCEV). ${ }^{28}$

Cross-sectional images were obtained using spectral-domain optical coherence tomography (SD-OCT; Heidelberg Engineering, Heidelberg, Germany) in 12 patients; a $20^{\circ} \times 15^{\circ}$ 19-line scan covering the fovea was used. Total retinal thickness, outer nuclear layer (ONL) thickness, and photoreceptor-RPE (PR+RPE) complex thickness were measured at the foveal dip and at 0.25-, 0.5-, 1-, 1.5-, 2-, and 2.5-mm eccentric distances using Heidelberg Eye Explorer software (Version 1.6.4.0; Heidelberg Engineering). Outer nuclear layer thickness 
was measured from the outer plexiform layer to the external limiting membrane (ELM); PR+RPE thickness was measured from the ELM to Bruch's membrane; and total retinal thickness was measured from the vitreous-retinal interface to RPEBruch's membrane complex. Clinically normal values for total retinal thickness, ONL thickness, and PR+RPE thickness were obtained from 25 age-matched individuals (mean age, 46 years; range, 27-62 years) with no retinal or vitreoretinal disease; we performed a postacquisition interpolation of the normal data using custom programs (MatLab R2011a; The MathWorks, Inc., Natick, MA, USA)

We acquired FAF images using a confocal scanning laser ophthalmoscope (cSLO; Spectralis; Heidelberg Engineering). After the pupil was dilated, $30^{\circ}$ and/or $55^{\circ}$ field-of-view FAF images were obtained from all patients (except case 11) using an optically pumped solid-state laser with 488-nm excitation. Two independent observers (RACvH and NMB) measured the size of the atrophic lesions (determined using the absence of autofluorescence) as described previously. ${ }^{29}$

\section{Results}

\section{Clinical Characteristics}

Of the 198 patients with a confirmed diagnosis of STGD1, 13 unrelated patients (7\%) had foveal sparing. The clinical characteristics of these 13 patients (including all 26 eyes) are summarized in Table 1 . This cohort included five women and eight men; all 13 patients were of Caucasian descent, with a mean age at onset of 52 years (range, 32-67 years). The mean disease duration was 9 years (range, 1-34 years). These patients were diagnosed between the age of 39 and 82 years (mean, 57 years). None of the patients were using hydroxychloroquine at the time of the study, nor had they used this drug in the past.

Foveal sparing was present in 17 of the 26 eyes (65\%), and five of the 13 patients (38\%) had bilateral foveal sparing. In seven of the patients with unilateral foveal sparing, five of the contralateral eyes had no signs of RPE atrophy or parafoveal atrophic RPE lesions that surrounded the fovea by less than $180^{\circ}$. In the remaining two eyes, and in both eyes in patient 12 , the fovea degenerated after initial foveal sparing (Table 1).

Nine of the 13 patients (69\%) initially experienced a decline in visual acuity. Other initial symptoms included paracentral scotoma in four patients (31\%), metamorphopsia in two patients (15\%), and nyctalopia in one patient (8\%). Two patients (patients 2 and 13;15\%) initially experienced no visual complaints, but were referred to our department because of fundus abnormalities found during ophthalmologic screening for glaucoma or thyroid eye disease; after 9 and 6 years, respectively, these two patients experienced a perceived decrease in visual acuity (Table 1 ). The BCVA was $20 / 40$ or better in all 17 eyes with foveal sparing; seven of these 17 eyes ( $41 \%$ ) had a BCVA of $20 / 25$ or better. In the four eyes in which atrophy ultimately affected the fovea, BCVA had decreased to $\leq 20 / 200$ from an initial acuity of $20 / 25$ to $20 / 40$ when the fovea was spared.

In 12 patients, a fundus examination revealed irregular flavimaculatus flecks scattered throughout the posterior pole and occasionally extending anterior to the vascular arcades (Figs. 2A, 2B, 3A). One patient had small perifoveal yellowwhite dots. Patient 12, who had bilateral foveal degeneration, developed extensive chorioretinal atrophy of the posterior pole and the midperiphery during the patient's 26 years of follow-up (Fig. 2C). Masking of the choroidal background fluorescence (i.e., a so-called dark choroid) was evident on FA imaging in seven patients, four of whom carried a single heterozygous $A B C A 4$ mutation (Tables 1, 2). The flavimaculatus flecks were visible as an irregular pattern of hyperfluorescence and hypofluorescence on FA imaging (Fig. 3B), and they appeared as hyperautofluorescent flecks on FAF imaging (Figs. 3C, 4). The chorioretinal atrophy corresponded with sharply delineated areas that included both an absence of autofluorescence and structural thinning of the outer retinal layers on OCT (Fig. 3D). Static perimetry revealed sharply delineated absolute parafoveal scotomas (Fig. 4) with subnormal foveal sensitivity (median, $35 \mathrm{~dB}$; range, 20-39 dB). Foveal sensitivity was not measurable $(<0 \mathrm{~dB})$ in patient 12 , which is consistent with the anatomical findings and other functional results obtained from this patient.

The results of the ffERG recordings in 10 eyes with foveal sparing are summarized in Table 1 . In these 10 eyes, photopic amplitude was normal in seven eyes (70\%) and moderately reduced in three eyes (30\%), and scotopic amplitude was normal in eight eyes (80\%) and moderately reduced in two eyes (20\%). In the four eyes that developed an atrophic fovea after initial foveal sparing, the photopic and scotopic amplitudes were either normal or severely reduced (Table 1). In three patients (patient 5, right eye; patient 8 , left eye; and patient 13 , left eye), the scotopic amplitude was reduced more markedly than the amplitude of photopic flash responses, although none of these patients had peripheral pigmentary retinopathy. No clear correlation was found between ffERG response and disease duration, visual acuity, or fundoscopic characteristics. We also measured mfERG in eight eyes with foveal sparing and found that the P1-response amplitudes in the central two rings (representing the foveal retina) were relatively intact compared to the outer three rings, which showed severely reduced responses.

\section{Retinal Structure}

Spectral-domain OCT imaging was performed in 12 patients and revealed highly localized damage to the lamellar architecture of the macula. We observed parafoveal atrophy of photoreceptors and RPE cells with sharp borders, represented by a loss of the bands associated with the ELM, the ellipsoid inner segments, and the RPE (Figs. 5B-5E). ${ }^{30}$ These bands were present in the fovea, however, although many of the bands that corresponded to the ELM and ellipsoid inner segments were irregular. In addition, the longer outer segments-which are characteristic of cone photoreceptors in the foveal dip-were not observed in any of these 12 eyes (Figs. 5A-5D). In three eyes, we observed signs reminiscent of outer retinal tubulation at the border of the atrophic lesions (Fig. 5D), although no rosette-like structures (as described by Zweifel and colleagues $^{31}$ ) were observed. Microcystoid macular edema was present in three eyes (Fig. 5C), and epiretinal gliosis with retinal wrinkling extending over the fovea was observed in one eye.

An analysis of the retinal lamellar architecture revealed that the overall retinal thickness in the foveal and parafoveal regions was generally decreased (Fig. 5F). The thickness of both the ONL and PR+RPE layers was normal at the foveal center in most patients. However, in the parafoveal region, the $\mathrm{PR}+\mathrm{RPE}$ was usually nearly absent, whereas the ONL was progressively thinner in this region (Fig. 5F).

\section{Natural Course of Foveal Sparing}

An analysis of the longitudinal FAF data, which were available for six patients, enabled us to investigate the natural course of foveal sparing. In general, fundus flavimaculatus flecks were observed in the early stages, in some cases even before symptoms developed. Within one to two decades of diagnosis 


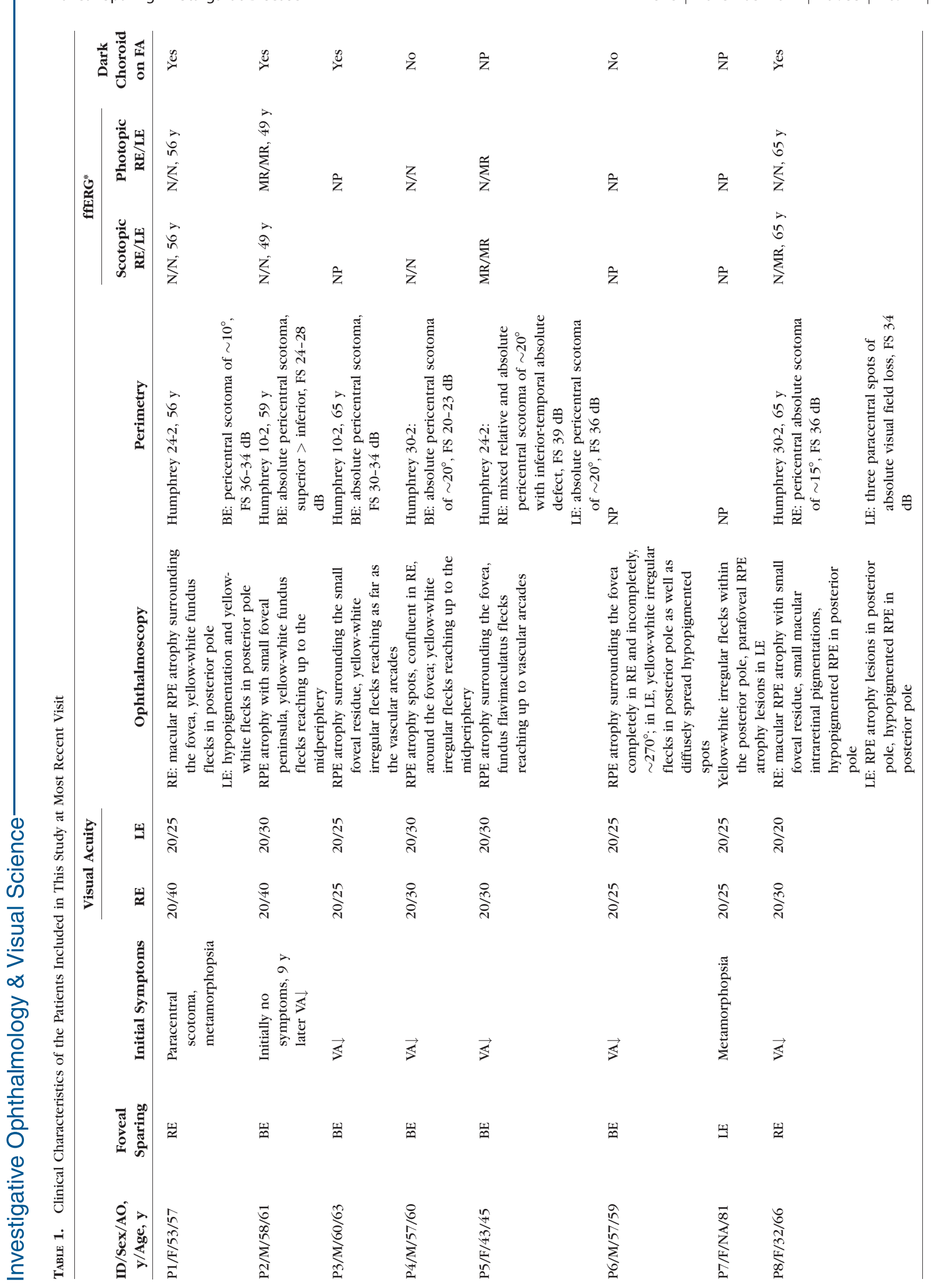




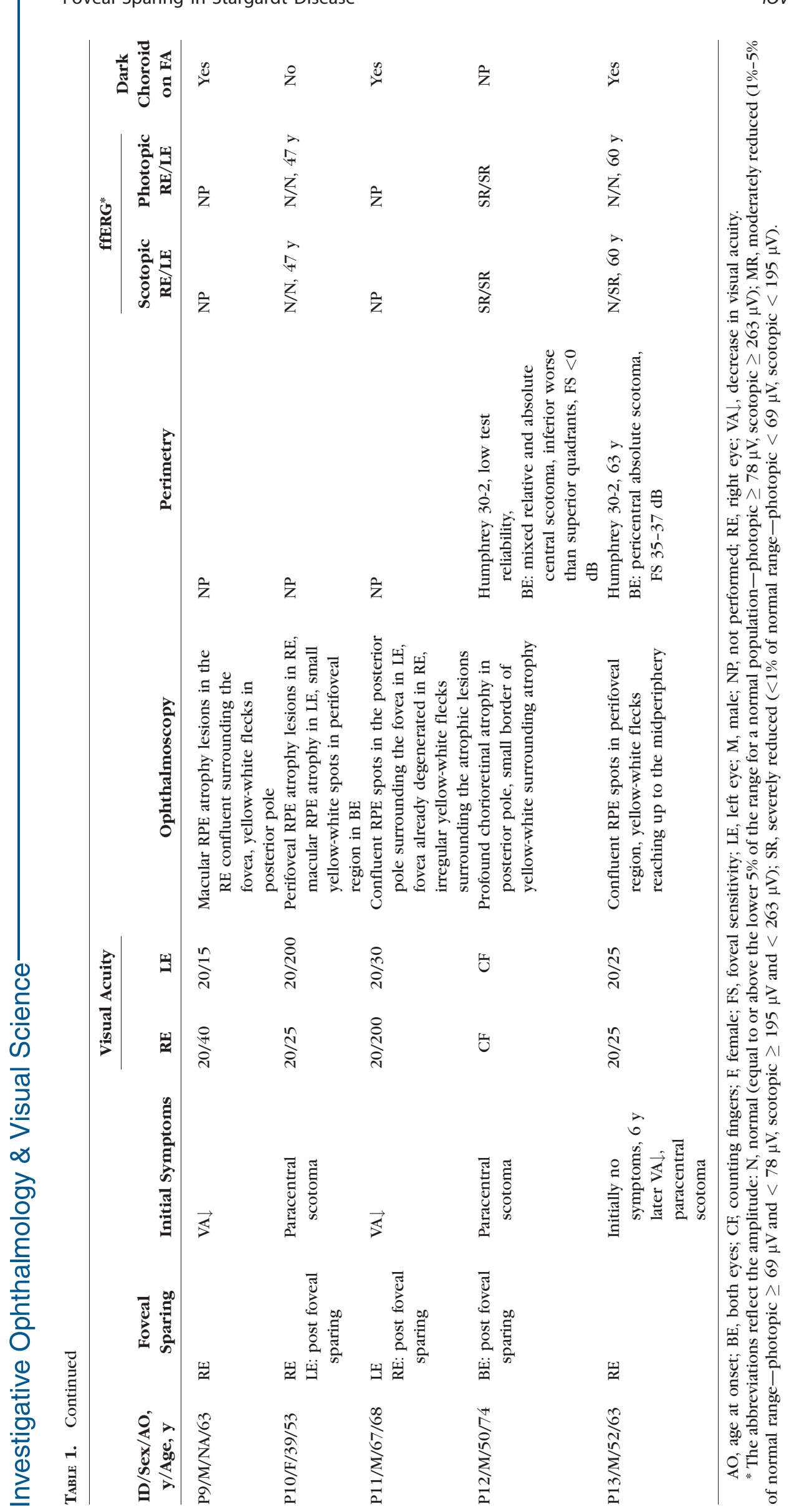



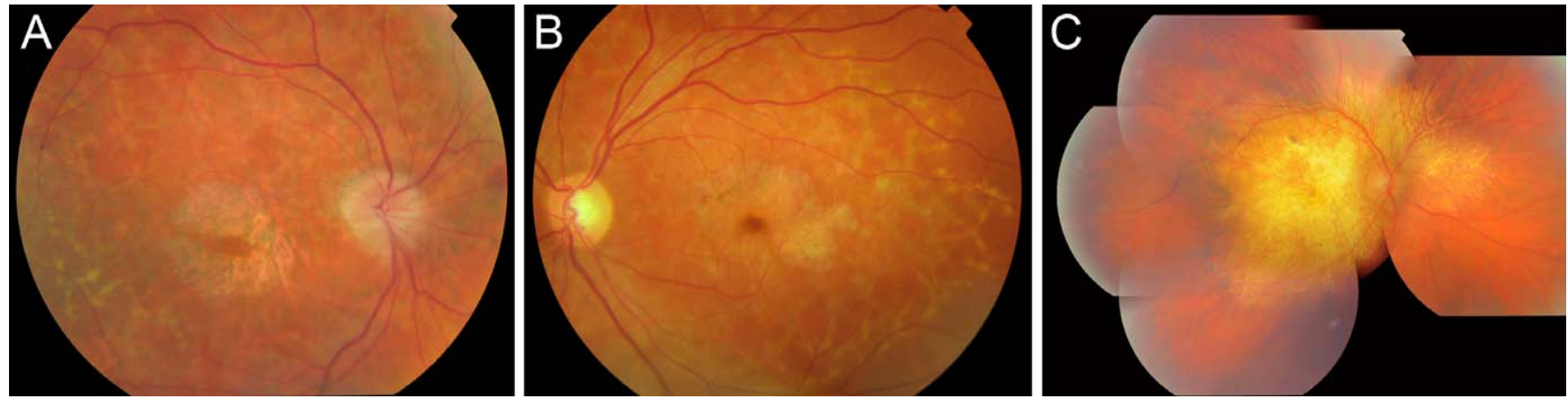

FigURE 2. Fundus photographs of three patients with STGD1. (A) Fundus photograph of the right eye of patient 4 (at age 60), showing fundus flavimaculatus flecks and parafoveal atrophy of the RPE. (B) Fundus photograph of the left eye of case 5 (at age 45), showing fundus flavimaculatus flecks and parafoveal atrophy of the RPE. (C) Mosaic of fundus photographs of the right eye of case 12 (at age 73), showing profound central atrophy of the RPE; this patient previously had foveal sparing.

(range, $10-18$ years), one or more sharply delineated parafoveal RPE atrophic areas appeared (Fig. 4A). Over time, these lesions expanded and reached confluence around the fovea, thus producing the foveal sparing phenotype. We observed three distinct stages in the development of foveal sparing; these stages characterize the natural course of the phenomenon. In stage 1, parafoveal atrophic lesions emerge, with several intact RPE connections between the fovea and the surrounding vital RPE (Figs. 4A, 4C). In stage 2, the atrophic RPE lesions interconnect, leaving only a single isthmus of RPE, thus resulting in a "peninsula-like" appear- ance (Figs. 4E. 4G). Stage 3 is characterized by an isolated fovea that is surrounded completely by RPE atrophy (Figs. 4I, $4 \mathrm{~K})$. We measured the expansion rate of the atrophic lesions in four patients using the FAF follow-up data (Table 3). The rate of expansion ranged from 0.832 to $2.363 \mathrm{~mm}^{2} / \mathrm{y}$ in these patients, and the rate was positively correlated to the size of the atrophic lesions. Given this wide range of expansion rates, it is unclear how long foveal sparing is present before the foveal structure and function become significantly affected by the profound atrophy; however, we observed that the foveal structure and function were relatively preserved for up to 6
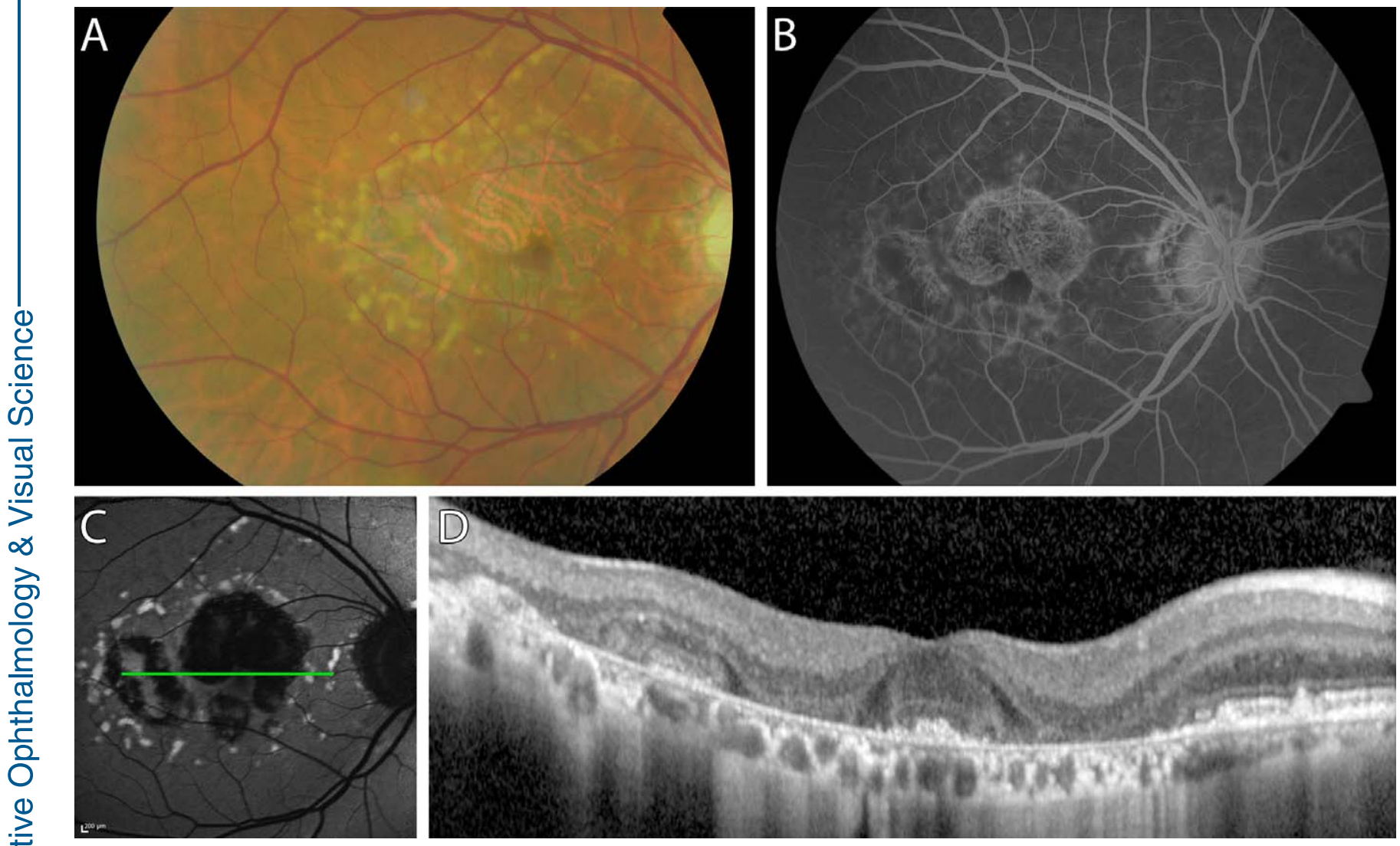

Figure 3. Multimodal imaging of the right fundus of patient 3 at age 62. (A) Fundus photograph showing irregular flavimaculatus flecks and atrophy of the central RPE; note that the fovea is spared. (B) Fluorescein angiograph showing the dark choroid sign, hyperfluorescence, visible choroidal vessels due to window defects, and a normal-appearing fovea. (C) Fundus autofluorescence image showing hyperautofluorescent flecks and RPE atrophy with foveal sparing. The green borizontal line indicates the scanning level of the OCT scan in (D). (D) An OCT scan through the fovea clearly shows the preserved cone photoreceptors in the fovea surrounded by atrophy of the outer retina and RPE. 
TABLe 2. ABCA4 Mutations in STGD1 Patients With Foveal Sparing

\begin{tabular}{|c|c|c|c|c|c|}
\hline & \multicolumn{2}{|c|}{ Allele 1} & \multicolumn{2}{|c|}{ Allele 2} & \multirow[b]{2}{*}{ References } \\
\hline & DNA Variant & Effect & DNA Variant & Effect & \\
\hline P1 & c. $5461-10 \mathrm{~T} \rightarrow \mathrm{C}$ & Unknown & NI & NA & 35,36 \\
\hline $\mathrm{P} 2$ & c. $3113 \mathrm{C} \rightarrow \mathrm{T}$ & p.Ala1038Val & c. $3874 \mathrm{C} \rightarrow \mathrm{T}$ & p.Gln $1292^{*}$ & $16,37,38,58$ \\
\hline $\mathrm{P} 3$ & c. $5461-10 \mathrm{~T} \rightarrow \mathrm{C}$ & Unknown & c. $5537 \mathrm{~T} \rightarrow \mathrm{C}$ & p.Ile1846Thr & $23,35,39,58$ \\
\hline P4 & c. $4363 \mathrm{~T} \rightarrow \mathrm{C}$ & p.Cys1455Arg & NI & NA & 40 \\
\hline P5 & c. $1822 \mathrm{~T} \rightarrow \mathrm{A}$ & p.Phe608Ile & c. $4685 \mathrm{~T} \rightarrow \mathrm{C}$ & p.Ile1562Thr & $23,40,41,59$ \\
\hline $\mathrm{P} 6$ & c. $768 \mathrm{G} \rightarrow \mathrm{T}$ & Splice defect & c. $3113 \mathrm{C} \rightarrow \mathrm{T}$ & p.Ala1038Val & $16,23,37$ \\
\hline P7 & c. $5196+1 \mathrm{G} \rightarrow \mathrm{T}$ & Splice defect & NI & NA & 45,58 \\
\hline P8 & c. $3874 \mathrm{C} \rightarrow \mathrm{T}$ & p.Gln $1292^{*}$ & NI & NA & 38 \\
\hline P9 & c. $5461-10 \mathrm{~T} \rightarrow \mathrm{C}$ & Unknown & NI & NA & 35,58 \\
\hline P10 & c. $1822 \mathrm{~T} \rightarrow \mathrm{A}$ & p.Phe608Ile & NI & NA & 23,41 \\
\hline P11 & c. $286 \mathrm{~A} \rightarrow \mathrm{G}$ & p.Asn96Asp & NI & NA & 43 \\
\hline P12 & c. $1805 \mathrm{G} \rightarrow \mathrm{A}$ & p.Arg602GIn & c. $4462 \mathrm{~T} \rightarrow \mathrm{C}$ & p.Cys1488Arg & $37,39,42-44$ \\
\hline $\mathrm{P} 13$ & c. $3874 \mathrm{C} \rightarrow \mathrm{T}$ & p. $G \ln 1292^{*}$ & c. $1928 \mathrm{~T} \rightarrow \mathrm{G}$ & p.Val643Gly & 38,45 \\
\hline
\end{tabular}

NI, not identified; NA, not applicable.

* Nonsense mutation.

years in patients 8 and 12. Eventually, the remaining foveal tissue became progressively smaller, and atrophy of the central fovea was observed in four eyes (in patients 10, 11, and 12 ; Figs. $4 \mathrm{~L}, 4 \mathrm{~N}, 5 \mathrm{E}$ ); this was accompanied by a decline in central vision to $20 / 200$ or worse.

\section{Genetic Analysis and Mutation Screening}

An examination of the pedigrees of the 13 unrelated patients with foveal sparing revealed a recessive inheritance pattern in five patients; the other eight patients appeared to be isolated cases. Mutations in the ABCA4 gene were identified in 19 of the 26 alleles $(73 \%$, Table 2$)$. We identified compound heterozygous mutations in six patients (46\%) and single heterozygous mutations in the other seven patients (54\%). In total, 13 different $A B C A 4$ variants were identified, including nine missense mutations, two splice site mutations, one nonsense mutation, and one synonymous variant that affects splicing. ${ }^{23}$ Each of these variants has been described previously (Table 2).

The mutational effects of five of the identified mutations have been reported previously (Supplementary Table S1). Although functional data regarding the effects of the other eight mutations is not available, we can speculate on the functional effect of the nonsense mutation (p.Gln1292*). Assuming that the resulting mRNA is not subject to nonsense-mediated decay, the resulting truncated protein will lack the second extracytoplasmic domain and the nucleotidebinding domain, which are involved in substrate and ATP binding, respectively. ${ }^{32}$ Supplementary Table S2 summarizes the identified missense mutations in $A B C A 4$, including allele frequencies in the exome variant server (EVS) and scores obtained from selected predictive tools. No differences in phenotype were observed between patients carrying severe mutations and patients carrying mild mutations. Finally, no pathogenic mutations in the PRPH2 gene were identified in this cohort, thereby excluding the possibility of pseudoStargardt pattern dystrophy. ${ }^{27}$

\section{Discussion}

Here, we present the clinical characteristics of 13 STGD1 patients with foveal sparing in one or both eyes. The majority of these patients were diagnosed with late-onset STGD1; only 3 patients developed symptoms prior to the age of 45 (Table 1).
In eyes with foveal sparing, visual acuity was relatively preserved; nevertheless, most of the patients experienced some loss of vision, which caused them to seek ophthalmologic care. In these instances, ophthalmoscopy readily revealed advanced retinal disease, with yellow-white irregular pisciform flecks and profound RPE atrophy adjacent to the fovea. Automated perimetry revealed perifoveal scotomas of various sizes with intact foveal sensibility. This combination of mild vision loss together with profound retinal abnormalities is typical among STGD1 patients with foveal sparing.

Recently, Fujinami et al. ${ }^{26}$ reported the clinical and molecular findings of a cohort of Stargardt patients with a foveal sparing phenotype. In their study, the authors included STGD1 patients with a functional fovea, irrespective of the presence of parafoveal RPE atrophy; thus, they studied a heterogeneous cohort of 40 patients. In contrast, in our cohort we defined foveal sparing as profound RPE atrophy that surrounded the fovea by at least $180^{\circ}$ and spared the fovea's structure and function. This clinical presentation is a rare finding among STGD1 patients, and our strict selection criterion resulted in a small but homogeneous cohort with a consistent phenotype and excluded STGD1 patients with lateonset disease that began with foveal atrophy. Moreover, our definition of foveal sparing is consistent with previously reported cases of foveal sparing in patients with other degenerative diseases. ${ }^{6,8,33,34}$ Despite the differences between our cohort and the cohort described by Fujinami et al., ${ }^{26}$ the visual acuity and electrophysiology findings in their paper are similar to the findings in our study; nevertheless, none of our patients were carriers of the $A B C A 4$ p.Arg2030Gln missense mutation, which was suggested previously to be prevalent among STGD1 patients with foveal sparing. ${ }^{26}$

\section{The Etiology of Foveal Sparing}

In our study, screening the $A B C A 4$ gene identified 19 pathogenic mutations that were described previously in STGD1 and/or other $A B C A 4$-associated retinopathies (Table 2) ${ }^{16,23,35-45}$; interestingly, however, foveal sparing was not described in any of the patients who were previously reported to carry these mutations. In a previously proposed model that links phenotype severity to the degree of residual ABCR function, ${ }^{23,24}$ late-onset STGD1 with foveal sparing was placed at the mild end of the spectrum of $A B C A 4$-associated retinopathies. ${ }^{3}$ Indeed, none of our STGD1 patients with foveal sparing had two $A B C A 4$ variants that are associated with 

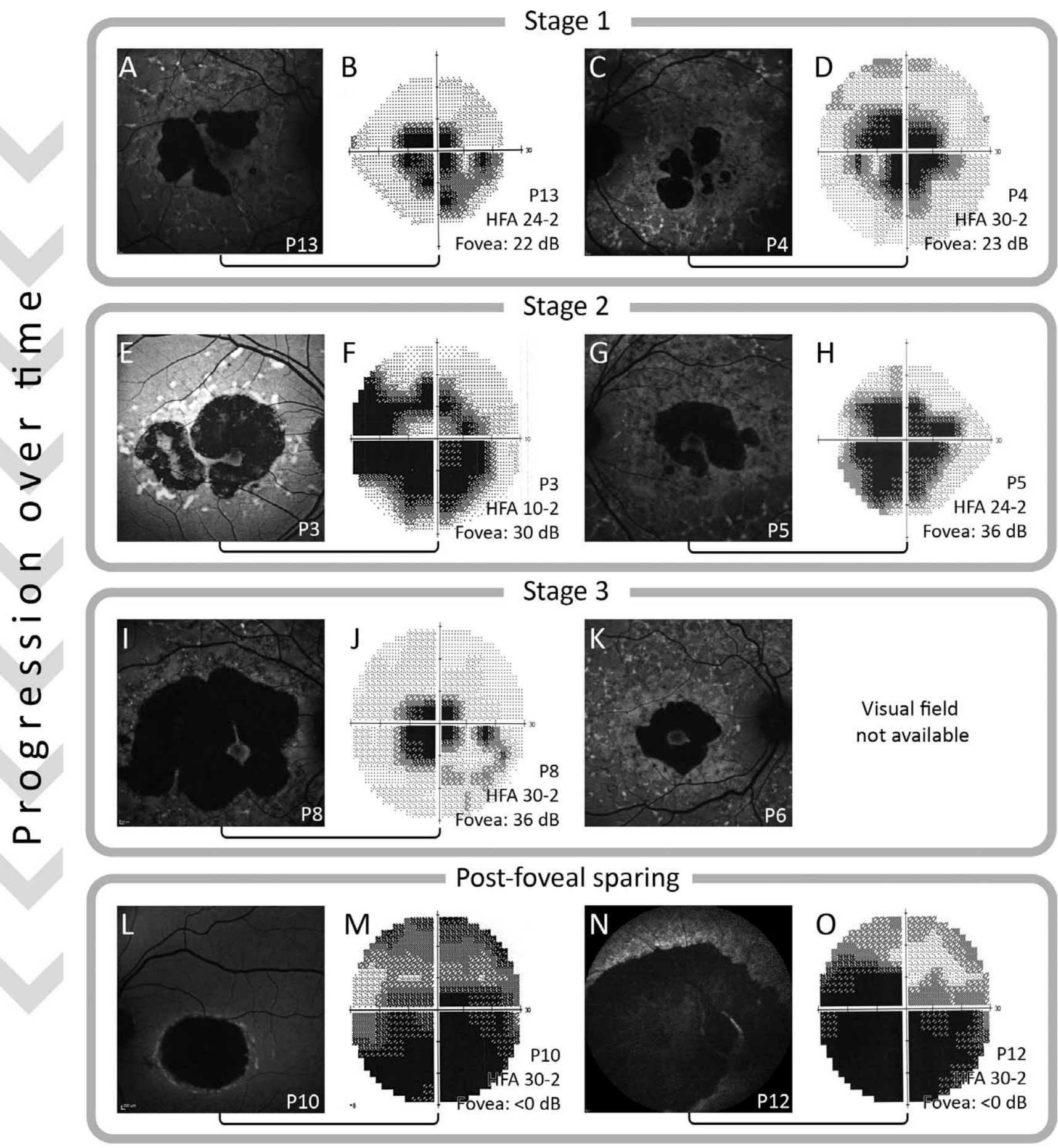

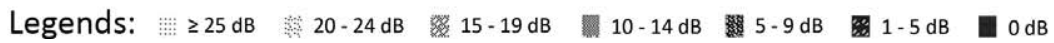

FIGURE 4. Natural course of fundus and perimetric changes in STGD1 with foveal sparing. FAF imaging can be used to identify the three stages that occur in foveal sparing. In stage 1, confluent parafoveal RPE lesions surround the macula, leaving several connections of intact RPE with the surrounding vital RPE (stage $1[\mathbf{A}, \mathbf{C}]$ ). Over time, the RPE atrophy progresses, and the lesions interconnect, leaving only one isthmus of RPE (stage 2 $[\mathbf{E}, \mathbf{G}]$ ). Further disease progression leads to an isolated fovea that is surrounded completely by RPE atrophy (stage $3[\mathbf{I}, \mathbf{K}]$ ). Eventually, RPE atrophy overcomes foveal resistance, leading to foveal degeneration (post foveal sparing $[\mathbf{L}, \mathbf{N}]$ ). Static perimetry examination reveals absolute perifoveal scotomas with intact foveal sensitivity in all eyes with foveal sparing $(\mathbf{B}, \mathbf{D}, \mathbf{F}, \mathbf{H}, \mathbf{J})$. Large absolute scotomas with diminished foveal sensitivity were observed in the eyes post foveal sparing (M, O). (A, B) FAF (A) and 24-2 perimetry (B) in patient 13 at age 63. (C, D) FAF (C) and 30-2 perimetry (D) in patient 4 at age 60 and 61, respectively. (E, F) FAF (E) and 10-2 perimetry (F) in patient 3 at age 64 and 65, respectively. (G, H) FAF (G) and 24-2 perimetry $(\mathbf{H})$ in patient 5 at age 45 . (I, J) FAF (I) and 30-2 perimetry (J) in patient 8 at age 66 and 65, respectively. (K) FAF in patient 6 at age 58 . (L, M) FAF (L) and 30-2 perimetry (M) in patient 10 at age 53. (N, O) FAF (N) and 30-2 perimetry (O) in patient 12 at age 73. 
A

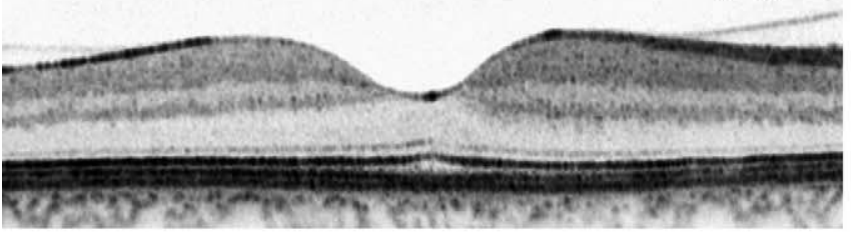

B

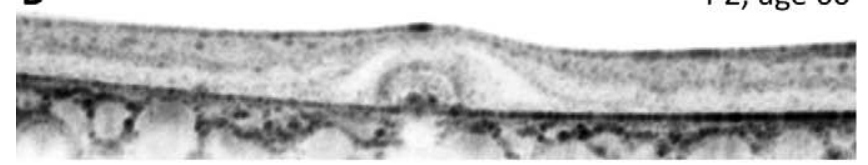

C

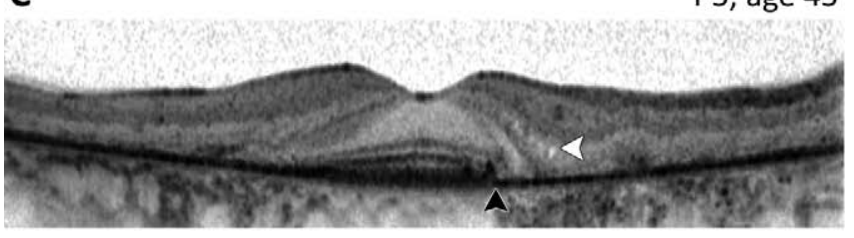

D

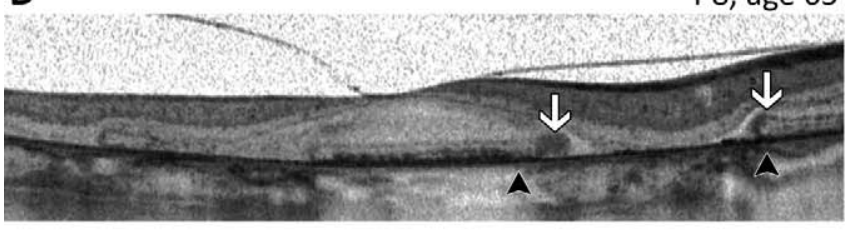

E

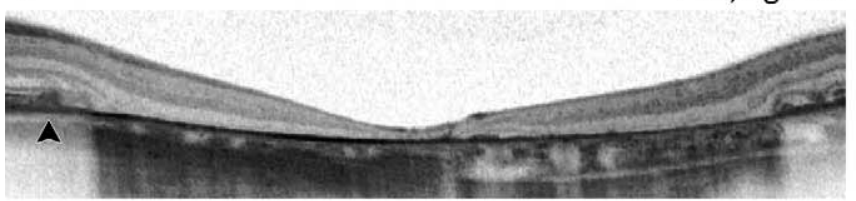

$F$
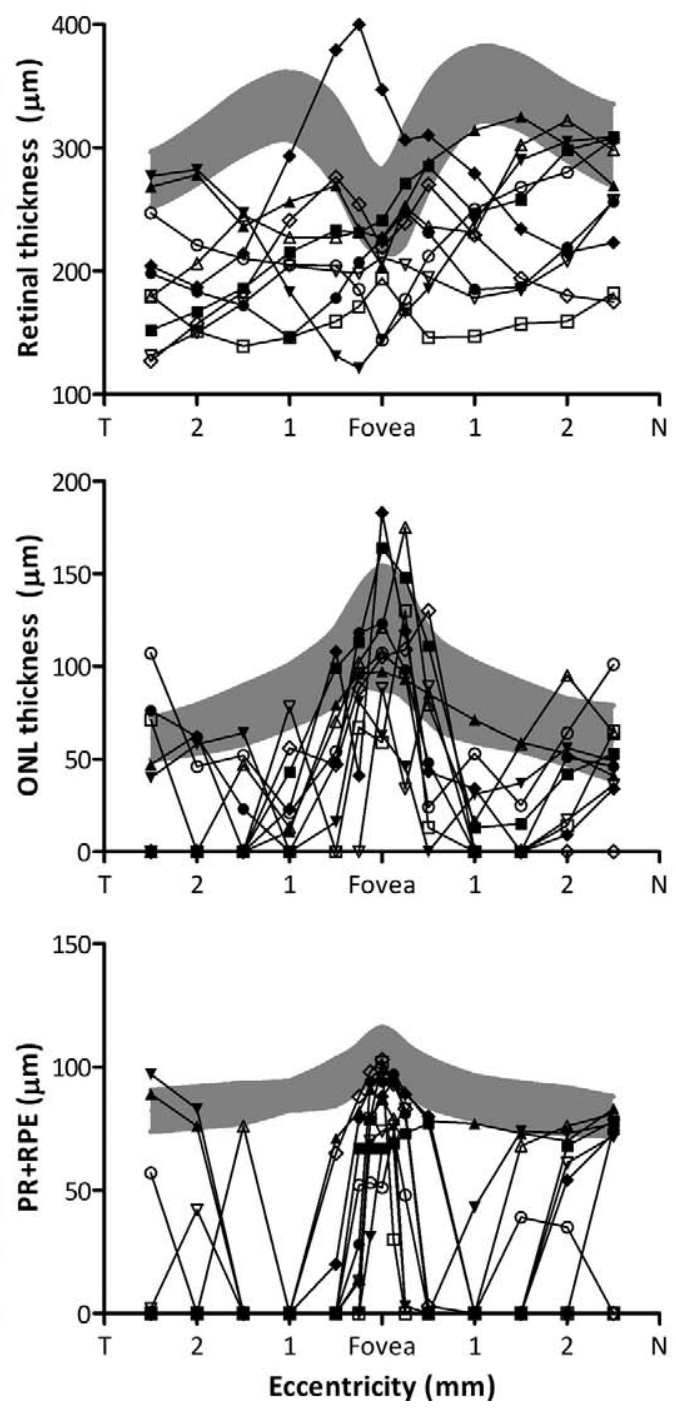

Healthy

$\rightarrow$ P1

๑- P2

$\triangle P 3$

$\rightarrow$ P4

$\multimap$ P5

$\rightarrow P 6$

$\rightarrow$ P8

$\leftarrow$ P9

$\rightarrow \mathrm{P} 10$

$\rightarrow$ P13

Figure 5. OCT analysis of Stargardt patients with foveal sparing. (A-E) The structural aspects of the macula in a healthy individual ([A] age 51), case 2 ([B] age 60), case 3 ([C] age 45), case 8 ([D] age 65), and case 10 ([E] age 53). The white arrows in (D) indicate the locations of signs resembling outer retinal tubulation. The black arrowheads in $(\mathbf{C}, \mathbf{D}, \mathbf{E})$ indicate the locations of abrupt photoreceptor layer disturbances without gradual outer and/or inner segment loss. The white arrowhead in (C) indicates the presence of microcysts in the inner nuclear layer. (F) Summary of thickness measurements of the total retina (top), ONL (middle), and PR+RPE (bottom). The gray shaded areas show the distribution (mean \pm 2 SD) of the total retinal, ONL, and PR+RPE thickness in 25 age-matched healthy individuals (mean age, 46 years).

a severe loss of ABCR function. However, our knowledge regarding the functional consequences of $A B C A 4$ mutations identified to date is limited (Supplementary Table S1). It can be extremely difficult to assess the effect of most missense variants using in silico predictions and allele frequencies in healthy individuals, for whom this information is often incomplete (Supplementary Table S2); in addition, assessing the combined effect of carrying two $A B C A 4$ variants is

TABLE 3. Results of Progression Analysis on Fundus Autofluorescence Imaging During Follow-Up in STGD1 Patients With Foveal Sparing

\begin{tabular}{|c|c|c|c|c|c|c|c|c|}
\hline \multirow[b]{2}{*}{ ID } & \multirow{2}{*}{$\begin{array}{c}\text { Age at Initial } \\
\text { Visit, } y\end{array}$} & \multicolumn{2}{|c|}{ Duration Follow-up } & \multirow[b]{2}{*}{ Eye } & \multirow{2}{*}{$\begin{array}{l}\text { Atrophy at Initial } \\
\text { Visit, } \mathbf{m m}^{2 *}\end{array}$} & \multirow{2}{*}{$\begin{array}{c}\text { Atrophy at } \\
\text { Follow-up, } \mathbf{m m}^{2 *}\end{array}$} & \multirow{2}{*}{$\begin{array}{l}\text { Expansion During } \\
\text { Follow-up, } \mathbf{m m}^{2 *}\end{array}$} & \multirow{2}{*}{$\begin{array}{c}\text { Progression Rate } \\
\mathbf{m m}^{2} / \mathbf{y}^{*}\end{array}$} \\
\hline & & Days & Years & & & & & \\
\hline P1 & 57 & 597 & 1.6 & Right & 3.517 & 4.877 & 1.36 & 0.832 \\
\hline \multirow[t]{2}{*}{$\mathrm{P} 2$} & 59 & 842 & 2.3 & Right & 11.792 & 15.770 & 3.978 & 1.726 \\
\hline & & & & Left & 11.202 & 16.43 & 5.228 & 2.268 \\
\hline P8 & 63 & 1657 & 4.5 & Right & 16.308 & 27.027 & 10.719 & 2.363 \\
\hline P9 & 62 & 420 & 1.1 & Right & 3.570 & 4.601 & 1.031 & 0.897 \\
\hline
\end{tabular}

* Measurements were performed by two authors (RACvH and NMB), and the average of the two measurements is shown. Mean interobserver variance was $2.7 \%$ (range, $0.4 \%-9.8 \%$ ). 
particularly difficult. Functional assays are needed in order to form definitive conclusions regarding the effects of these mutations.

Because foveal sparing can be present in phenotypes that are independent of $A B C A 4$ mutations, including AMD and mitochondrial retinal dystrophy, $6,8-12,17,20,25,44,45$ genetic factors other than $A B C A 4$ mutations are likely involved. These factors could include single nucleotide polymorphisms (SNPs) and even mutations in retina-specific genes other than $A B C A 4$, which suggests that a digenic or triallellic trait-in combination with the identified $A B C A 4$ mutations-may underlie the degenerative pattern observed in our patients. Moreover, anatomical, metabolic, and/or biochemical factors may underlie foveal sparing. For example, the average peak density of cones in the fovea is 199,000 cones $/ \mathrm{mm}^{2}$, but can range from 98,200 to 324,100 cones $/ \mathrm{mm}^{2}{ }^{2}$ The initial number of cones in the fovea may play a role in the development of foveal sparing; however, adaptive optics imaging techniques-which can provide the resolution needed to determine photoreceptor density in vivo-are not generally available in most ophthalmology practices. Another factor to consider is that S ("blue") cone photoreceptors, which are absent in the foveal center, seem to be more vulnerable to retinal disease than $\mathrm{M}$ and $\mathrm{L}$ cones, although this selective vulnerability has not been reported in STGD1. ${ }^{46,47}$ Moreover, parafoveal rods appear to be more vulnerable than cones to the effects of aging and alltrans-retinal-mediated damage. ${ }^{48-50}$ This difference may arise from the sole dependence of rods on the RPE for replenishing 11-cis-retinal; in contrast, cones are also supplied by Müller cells. ${ }^{51}$ Furthermore, cone cells have a slower turnover rate of outer segments compared to rods, ${ }^{52}$ although this does not necessarily result in higher all-trans-retinal levels in RPE cells, as regeneration is faster in cones than in rods. ${ }^{51}$ Macular pigments, which can filter out high-energy light, may also serve a protective role, given that light exposure is crucial in the pathogenesis of STGD1. ${ }^{53,54}$ In addition, rod-derived cone viability factor (RdCVF), which is believed to prevent cone degeneration, ${ }^{55}$ may also play a role. Importantly, the absolute levels of macular pigments and RdCVF may differ between STGD1 patients with foveal sparing and STGD1 patients without foveal sparing.

\section{Differential Diagnosis and Clinical Significance of Foveal Sparing}

When forming a diagnosis, foveal sparing-associated clinical entities other than late-onset STGD should be considered, including geographic atrophy in AMD, mitochondrial retinal dystrophy associated with the $\mathrm{m} .3243 \mathrm{~A}>\mathrm{G}$ mutation, central areolar choroidal dystrophy, and pseudo-Stargardt pattern dystrophy. 6,9,10,27,29 Importantly, misdiagnosing this condition can lead to inappropriate genetic counseling (these diseases display unique inheritance patterns) and/or an inaccurate estimate of the prognosis. Furthermore, in the event of an incorrect diagnosis of AMD, prescribing vitamin A-rich nutritional supplements can accelerate the accumulation of all-trans-retinal-derived toxins and increase the rate of disease progression, as shown in the retinas of homozygous Abca4knockout mice. ${ }^{56}$ Stargardt disease with foveal sparing can be diagnosed based on the presence of characteristic pisciform flecks together with RPE atrophy surrounding the fovea, a "dark choroid" sign on FA, and genetic analysis of the $A B C A 4$ gene. Fundus autofluorescence imaging can clearly highlight the fundus flecks, which appear as hyperautofluorescent flecks, and RPE atrophy, which appears as an absence of autofluorescence. Retinal dystrophies that closely resemble STGD1 can follow other patterns of inheritance-for example, due to mutations in mitochondrial DNA-or can be autosomal dominant, with variable penetrance and expression. The fact that the dark choroid sign is present in approximately $85 \%$ of patients with STGD1 $^{57}$ suggests a pivotal role for genetic analysis in the diagnosis of retinal dystrophies.

In conclusion, foveal sparing is a clinical phenomenon that occurs primarily in patients with late-onset STGD1 and is associated with the relative preservation of visual acuity, although visual acuity ultimately deteriorates by the end stage of the disease. Stargardt disease patients with foveal sparing may be promising candidates for future therapeutic trials, as delayed degeneration of the fovea increases the time window for applying therapeutic interventions such as gene therapy. Although the mechanisms that underlie foveal sparing are currently unclear, expanding our knowledge of the metabolic and biochemical processes that lead to foveal sparing can facilitate the development of therapeutic strategies aimed at preserving foveal function.

\section{Acknowledgments}

We thank all of the patients for their participation in this study. Additionally, we thank Michel M. Teussink, MSc, for his assistance with MathLab. We also thank Odette A.M. Tigchelaar-Besling, MD, for sharing her clinical observations.

Supported by the Stichting A.F. Deutman Researchfonds Oogheelkunde, Nijmegen, The Netherlands; Foundation Fighting Blindness USA (FFB Grants C-GE-0811-0545-RAD01 and BR-GE-0510-0489RAD); The Netherlands Organization for Health Research and Development (ZonMW; TOP-Grant 40-00812-98-09047); and the Nederlandse Oogonderzoek Stichting (NOS), Nijmegen, The Netherlands. CJFB was supported by a Niels Stensen Fellowship Award. The authors alone are responsible for the content and writing of the paper.

Disclosure: R.A.C. van Huet, None; N.M. Bax, None; S.C. Westeneng-Van Haaften, None; M. Muhamad, None; M.N. Zonneveld-Vrieling, None; L.H. Hoefsloot, None; F.P.M. Cremers, None; C.J.F. Boon, None; B.J. Klevering, None; C.B. Hoyng, None

\section{References}

1. Curcio CA, Sloan KR Jr, Packer O, Hendrickson AE, Kalina RE. Distribution of cones in human and monkey retina: individual variability and radial asymmetry. Science. 1987;236:579-582.

2. Curcio CA, Sloan KR, Kalina RE, Hendrickson AE. Human photoreceptor topography. J Comp Neurol. 1990;292:497523.

3. Westeneng-van Haaften SC, Boon CJ, Cremers FP, Hoefsloot LH, den Hollander AI, Hoyng CB. Clinical and genetic characteristics of late-onset Stargardt's disease. Ophthalmology. 2012;119:1199-1210.

4. Rotenstreich Y, Fishman GA, Anderson RJ. Visual acuity loss and clinical observations in a large series of patients with Stargardt disease. Ophthalmology. 2003;110:1151-1158.

5. Nakao T, Tsujikawa M, Sawa M, Gomi F, Nishida K. Foveal sparing in patients with Japanese Stargardt's disease and good visual acuity. Jpn J Ophthalmol. 2012;56:584-588.

6. de Laat P, Smeitink JA, Janssen MC, Keunen JE, Boon CJ. Mitochondrial retinal dystrophy associated with the m.3243A > G mutation. Ophthalmology. 2013;120:2684-2696.

7. Baker CI, Dilks DD, Peli E, Kanwisher N. Reorganization of visual processing in macular degeneration: replication and clues about the role of foveal loss. Vision Res. 2008; 48:19101919.

8. Forte R, Querques G, Querques L, Leveziel N, Benhamou N, Souied EH. Multimodal evaluation of foveal sparing in patients with geographic atrophy due to age-related macular degeneration. Retina. 2013;33:482-489. 
9. Mones J, Biarnes M, Trindade F, Arias L, Alonso J. Optical coherence tomography assessment of apparent foveal swelling in patients with foveal sparing secondary to geographic atrophy. Ophthalmology. 2013;120:829-836.

10. Schmitz-Valckenberg S, Fleckenstein M, Helb HM, Charbel Issa P, Scholl HP, Holz FG. In vivo imaging of foveal sparing in geographic atrophy secondary to age-related macular degeneration. Invest Ophthalmol Vis Sci. 2009;50:3915-3921.

11. Sunness JS, Rubin GS, Zuckerbrod A, Applegate CA. Fovealsparing scotomas in advanced dry age-related macular degeneration. J Vis Impair Blind. 2008;102:600-610.

12. Stargardt K. Über familliäre, progressive Degeneration in der Maculagegend des Auges. Graefes Arch Clin Exp Ophthalmol. 1909;534-550.

13. Fishman GA, Stone EM, Grover S, Derlacki DJ, Haines HL, Hockey RR. Variation of clinical expression in patients with Stargardt dystrophy and sequence variations in the ABCR gene. Arch Ophthalmol. 1999;117:504-510.

14. Lois N, Holder GE, Bunce C, Fitzke FW, Bird AC. Phenotypic subtypes of Stargardt macular dystrophy-fundus flavimaculatus. Arch Ophthalmol. 2001;119:359-369.

15. Lois N, Holder GE, Fitzke FW, Plant C, Bird AC. Intrafamilial variation of phenotype in Stargardt macular dystrophy-fundus flavimaculatus. Invest Ophthalmol Vis Sci. 1999;40:26682675.

16. Allikmets R, Singh N, Sun H, et al. A photoreceptor cellspecific ATP-binding transporter gene (ABCR) is mutated in recessive Stargardt macular dystrophy. Nat Genet. 1997;15: 236-246.

17. Molday LL, Rabin AR, Molday RS. ABCR expression in foveal cone photoreceptors and its role in Stargardt macular dystrophy. Nat Genet. 2000;25:257-258.

18. Sparrow JR, Wu Y, Kim CY, Zhou J. Phospholipid meets alltrans-retinal: the making of RPE bisretinoids. J Lipid Res. 2010; 51:247-261.

19. Cremers FP, van de Pol DJ, van Driel M, et al. Autosomal recessive retinitis pigmentosa and cone-rod dystrophy caused by splice site mutations in the Stargardt's disease gene ABCR. Hum Mol Genet. 1998;7:355-362.

20. Birch DG, Peters AY, Locke KL, Spencer R, Megarity CF, Travis GH. Visual function in patients with cone-rod dystrophy (CRD) associated with mutations in the ABCA4(ABCR) gene. Exp Eye Res. 2001;73:877-886.

21. Klevering BJ, Yzer S, Rohrschneider K, et al. Microarray-based mutation analysis of the ABCA4 (ABCR) gene in autosomal recessive cone-rod dystrophy and retinitis pigmentosa. Eur J Hum Genet. 2004;12:1024-1032.

22. Klevering BJ, Maugeri A, Wagner A, et al. Three families displaying the combination of Stargardt's disease with conerod dystrophy or retinitis pigmentosa. Ophthalmology. 2004; 111:546-553.

23. Maugeri A, van Driel MA, van de Pol DJ, et al. The 2588G->C mutation in the ABCR gene is a mild frequent founder mutation in the Western European population and allows the classification of ABCR mutations in patients with Stargardt disease. Am J Hum Genet. 1999;64:1024-1035.

24. van Driel MA, Maugeri A, Klevering BJ, Hoyng CB, Cremers FP. ABCR unites what ophthalmologists divide(s). Ophthalmic Genet. 1998;19:117-122.

25. Klevering BJ, Deutman AF, Maugeri A, Cremers FP, Hoyng CB. The spectrum of retinal phenotypes caused by mutations in the ABCA4 gene. Graefes Arch Clin Exp Ophthalmol. 2005; 243:90-100.

26. Fujinami K, Sergouniotis PI, Davidson AE, et al. Clinical and molecular analysis of stargardt disease with preserved foveal structure and function. Am J Ophthalmol. 2013;156:487-501, e481.
27. Boon CJ, van Schooneveld MJ, den Hollander AI, et al. Mutations in the peripherin/RDS gene are an important cause of multifocal pattern dystrophy simulating STGD1/fundus flavimaculatus. Br J Ophthalmol. 2007;91:1504-1511.

28. Marmor MF, Fulton AB, Holder GE, et al. ISCEV Standard for full-field clinical electroretinography (2008 update). Doc Ophthalmol. 2009;118:69-77.

29. Boon CJ, Klevering BJ, Cremers FP, et al. Central areolar choroidal dystrophy. Ophthalmology. 2009;116:771-782, 782.e 771 .

30. Spaide RF, Curcio CA. Anatomical correlates to the bands seen in the outer retina by optical coherence tomography: literature review and model. Retina. 2011;31:1609-1619.

31. Zweifel SA, Engelbert M, Laud K, Margolis R, Spaide RF, Freund KB. Outer retinal tubulation: a novel optical coherence tomography finding. Arch Ophthalmol. 2009;127:1596-1602.

32. Tsybovsky Y, Molday RS, Palczewski K. The ATP-binding cassette transporter ABCA4: structural and functional properties and role in retinal disease. Adv Exp Med Biol. 2010;703: 105-125.

33. Sunness JS, Bressler NM, Maguire MG. Scanning laser ophthalmoscopic analysis of the pattern of visual loss in agerelated geographic atrophy of the macula. Am J Ophthalmol. 1995;119:143-151.

34. Rath PP, Jenkins S, Michaelides M, et al. Characterisation of the macular dystrophy in patients with the A3243G mitochondrial DNA point mutation with fundus autofluorescence. $\mathrm{Br} \mathrm{J}$ Ophthalmol. 2008;92:623-629.

35. Maugeri A, Klevering BJ, Rohrschneider K, et al. Mutations in the ABCA4 (ABCR) gene are the major cause of autosomal recessive cone-rod dystrophy. Am J Hum Genet. 2000;67:960966.

36. Roberts LJ, Nossek CA, Greenberg LJ, Ramesar RS. Stargardt macular dystrophy: common ABCA4 mutations in South Africa-establishment of a rapid genetic test and relating risk to patients. Mol Vis. 2012;18:280-289.

37. Sun H, Smallwood PM, Nathans J. Biochemical defects in ABCR protein variants associated with human retinopathies. Nat Genet. 2000;26:242-246.

38. Ernest PJ, Boon CJ, Klevering BJ, Hoefsloot LH, Hoyng CB. Outcome of ABCA4 microarray screening in routine clinical practice. Mol Vis. 2009;15:2841-2847.

39. Webster AR, Heon E, Lotery AJ, et al. An analysis of allelic variation in the ABCA4 gene. Invest Ophthalmol Vis Sci. 2001; 42:1179-1189.

40. Rosenberg T, Klie F, Garred P, Schwartz M. N965S is a common ABCA4 variant in Stargardt-related retinopathies in the Danish population. Mol Vis. 2007;13:1962-1969.

41. Lewis RA, Shroyer NF, Singh N, et al. Genotype/Phenotype analysis of a photoreceptor-specific ATP-binding cassette transporter gene, ABCR, in Stargardt disease. Am J Hum Genet. 1999;64:422-434.

42. Wiszniewski W, Zaremba CM, Yatsenko AN, et al. ABCA4 mutations causing mislocalization are found frequently in patients with severe retinal dystrophies. Hum Mol Genet. 2005;14:2769-2778.

43. Papaioannou M, Ocaka L, Bessant D, et al. An analysis of ABCR mutations in British patients with recessive retinal dystrophies. Invest Ophthalmol Vis Sci. 2000;41:16-19.

44. Biswas-Fiss EE, Kurpad DS, Joshi K, Biswas SB. Interaction of extracellular domain 2 of the human retina-specific ATPbinding cassette transporter (ABCA4) with all-trans-retinal. $J$ Biol Chem. 2010;285:19372-19383.

45. Allikmets R, Shroyer NF, Singh N, et al. Mutation of the Stargardt disease gene (ABCR) in age-related macular degeneration. Science. 1997;277:1805-1807. 
46. Pokorny JSV, Verriest G, Pinckers A. Congenital and Acquired Color Vision Defects. New York: Grune \& Stratton; 1979.

47. Greenstein VC, Hood DC, Ritch R, Steinberger D, Carr RE. S (blue) cone pathway vulnerability in retinitis pigmentosa, diabetes and glaucoma. Invest Ophthalmol Vis Sci. 1989;30: 1732-1737.

48. Curcio CA. Photoreceptor topography in ageing and agerelated maculopathy. Eye (Lond). 2001;15:376-383.

49. Curcio CA, Millican CL, Allen KA, Kalina RE. Aging of the human photoreceptor mosaic: evidence for selective vulnerability of rods in central retina. Invest Ophthalmol Vis Sci. 1993;34:3278-3296.

50. Okano K, Maeda A, Chen $\mathrm{Y}$, et al. Retinal cone and rod photoreceptor cells exhibit differential susceptibility to lightinduced damage. J Neurochem. 2012;121:146-156.

51. Wang JS, Kefalov VJ. The cone-specific visual cycle. Prog Retin Eye Res. 2011;30:115-128.

52. Anderson DH, Fisher SK, Erickson PA, Tabor GA. Rod and cone disc shedding in the rhesus monkey retina: a quantitative study. Exp Eye Res. 1980;30:559-574.

53. Weiter JJ, Delori F, Dorey CK. Central sparing in annular macular degeneration. Am J Ophthalmol. 1988;106:286-292.
54. Aleman TS, Cideciyan AV, Windsor EA, et al. Macular pigment and lutein supplementation in ABCA4-associated retinal degenerations. Invest Ophthalmol Vis Sci. 2007;48:13191329.

55. Leveillard T, Mohand-Said S, Lorentz O, et al. Identification and characterization of rod-derived cone viability factor. Nat Genet. 2004;36:755-759.

56. Radu RA, Yuan Q, Hu J, et al. Accelerated accumulation of lipofuscin pigments in the RPE of a mouse model for ABCA4mediated retinal dystrophies following Vitamin A supplementation. Invest Ophthalmol Vis Sci. 2008;49:3821-3829.

57. Fishman GA, Farber M, Patel BS, Derlacki DJ. Visual acuity loss in patients with Stargardt's macular dystrophy. Ophthalmology. 1987;94:809-814.

58. Kitiratschky VB, Grau T, Bernd A, et al. ABCA4 gene analysis in patients with autosomal recessive cone and cone rod dystrophies. Eur J Hum Genet. 2008;16:812-819.

59. Rivera A, White K, Stohr H, et al. A comprehensive survey of sequence variation in the ABCA4 (ABCR) gene in Stargardt disease and age-related macular degeneration. Am J Hum Genet. 2000;67:800-813. 